\title{
How Can We Optimize the Granulose Cell Function of Oocytes from an Endometriosis Patient to Improve the Reproductive Outcomes-A Short Communication?
}

ISSN: 2640-9666

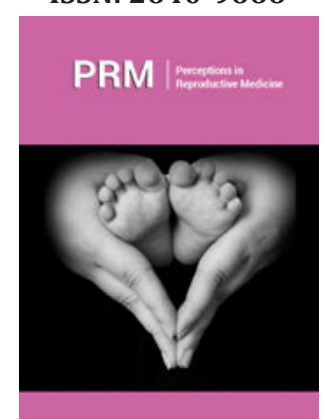

*Corresponding author: Kulvinder Kochar Kaur, Centre for Human Reproduction, India

Submission: 洒 January 17, 2020

Published: 㘹January 22, 2020

Volume 3 - Issue 4

How to cite this article: Kulvinder Kochar K, Gautam A, Mandeep S. How Can We Optimize the Granulose Cell Function of Oocytes from an Endometriosis Patient to Improve the Reproductive Outcomes-A Short Communication?. Perception in Reproductive Medicine.3(4). PRM.000570.2020

DOI: $10.31031 /$ PRM.2020.03.000570

Copyright@ Kulvinder Kochar Kaur, This article is distributed under the terms of the Creative Commons Attribution 4.0 International License, which permits unrestricted use and redistribution provided that the original author and source are credited.

\author{
Kulvinder Kochar Kaur*1, Gautam Allahbadia ${ }^{2}$ and Mandeep Singh ${ }^{3}$ \\ ${ }^{1,2}$ Centre for Human Reproduction, India \\ ${ }^{3}$ Department of Neurologist, India
}

\begin{abstract}
Endometriosis, results in infertility in 50\% infertile women. Explanations given are poor oocyte quality as well as low grade embryos associated with abnormal folliculogenesis, with reduced chances of fertilization correlated with enhanced oxidative stress, high reactive oxygen species (ROS) levels/local inflammation. Particularly escalated amounts of cytokines are found in follicular fluid of follicles of Endometriosis subjects. Here we further detail on the work going on in finding the role of granulosa cells in Endometriosis subjects with regards to increased inflammation, alteration in cytokines in follicular fluid that associates with follicular fluid in causing the oxidative stress, and how the upstream pathway involving tumour necrosis factor alpha $(\mathrm{TNF} \alpha)$ and nuclear factor kappa $\mathrm{B}(\mathrm{NF \kappa B})$ that influences both telomere length as well as telomerase activity, with shorter telomeres as well as reduced telomerase activity associated with aging follicles as well as those observed in patients with premature ovarian insufficiency (POI). Thus, this enhanced granulosa cell NFKB corresponds with inflammation found in FF along with effect on telomeres. Ways of targeting it seems to be one answer in improving reproductive potential of Endometriosis patients.
\end{abstract}

Keywords: Endometriosis; Poor oocyte quality; Oxidative stress; High reactive oxygen species (ROS); NFKB; TNF $\alpha$; Telomere length; Telomerase activity; POI

\section{Introduction}

Endometriosis, results in infertility in 50\% infertile women [1]. Explanations given are poor oocyte quality as well as low grade embryos associated with abnormal folliculogenesis [2], with reduced chances of fertilization correlated with enhanced oxidative stress, high reactive oxygen species (ROS) levels/local inflammation [3]. Particularly escalated amounts of cytokines are found in follicular fluid (FF) of patients with Endometriosis might result in ovulatory impairment [4]. Further Akila et al. reviewed how in the pathogenesis of ovarian pathologies like Endometriosis and polycystic ovarian syndrome (PCOS) are affected by enhanced ROS, enhanced oxidative stress in the ovary by concentrating on mural granulosalutein cells of in vitro Fertilization (IVF) subjects. Synthesis of anti-oxidant enzymes like peroxiredoxin 4, superoxide dismutase and catalase and oxidative stress damage response proteins like aldehyde dehydrogenase 3, member A2 reduces with aging in human granulosa lutein cells, that helps in an unbalance in ROS/anti-oxidants which modulate molecular injury as well as changes in cellular functioning. Increased oxidative stress in the granulosa cells associates with reduced expression of follicular stimulating hormone( FSH) receptor (FSHR) as well as a dysregulated FSHR signalling pathway and thus might have a role in impaired steroidogenic function as well as bad ovarian response to FSH in women who are aging. In case of women having Endometriosis along with those with PCOS have<antioxidants producing ability which may result in abnormal follicle formation as well as infertility. More investigations of the signalling pathways in relation to cellular responses to oxidative stress might throw light into molecular properties of these diseases and allow formation of new treating strategies for increasing reproductive potential in these women. Kunitomi et al. [5] posited that endoplasmic reticulum (ER) Stress is stimulated by great oxidative stress in granulose cells in ovaries from Endometrioma and this modulates oxidative stress-stimulated apoptosis. 
Human granulosa-lutein cells (GLC's) from Endometrioma patients expressed great mRNA amounts correlated with unfolded protein response (UPR). Additionally, amounts of phosphorylated ER Stress sensor proteins, inositol-requiring enzyme (IRE1) and double stranded RNA-activated protein kinase-like ER kinase (PERK), were enhanced in granulosa cells from Endometrioma patients. Knowing that ER Stress causes phosphorylation of ER Stress sensor proteins and stimulates UPR, these observations point that these cells were under ER Stress. $\mathrm{H}_{2} \mathrm{O}_{2}$, that stimulates oxidative stress, enhanced expression of UPR-related mRNA in cultured human GLC's with this action getting ameliorated by pre-treatment utilizing tauroursodeoxycholic acid (TUDCA), that is an ER Stress inhibitor utilized clinically. Therapy with $\mathrm{H}_{2} \mathrm{O}_{2}$ enhanced apoptosis as well as action of pro- apoptotic factors caspase 8 as well as caspase 3 both of which got ameliorated by TUDCA. This pointed that stimulated ER Stress stimulated by oxidative stress in GCs in ovaries having Endometrioma modulates apoptosis of these cells causing ovarian dysfunction in Endometriosis patients [6].

Reviewing the quality of oocytes in relation to endometriosis, Sanchez et al. [7] tried to concentrate on direct oocytes studied instead of most studies concentrating on cumulus cells or FF content. In summary It has been posited that morphological defects seen that include i)cytoplasmic granularity as well as/or presence of vacuoles might affect fertilization although limited predictive value of these findings in view of restrictions of non-invasive techniques with simple transmitted light microscopy along with subjectivity in morphological analysis as well as morphology might be influenced by other factors like ovarian stimulation or hormonal milieu. Goud et al did functional studies examining immature oocytes obtained from Endometriosis women as compared to control. They observed that oocytes from Endometriosis women showed enhanced cortical granule loss as well as ii) zona pellucida (ZP) hardening, probably interfering with fertilization dissolution of the $\mathrm{ZP}$ and the ability of embryo to undergo hatching and implantation (Figure1); $[8,9]$.

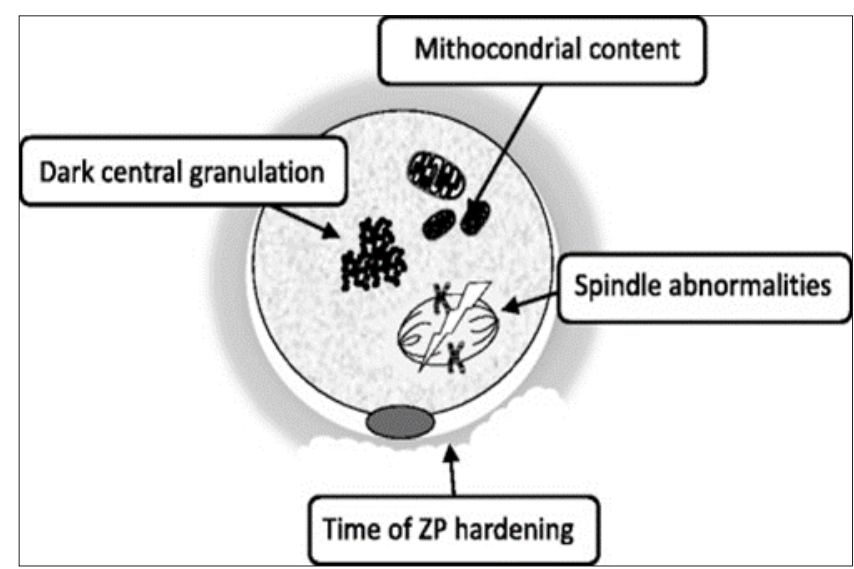

Figure 1: Courtesy ref no.-7-Representative morphological changes in oocytes from women affected by endometriosis.

Further capacity of oocytes to undergo in vitro maturation (IVM) to metaphase II stage was checked, observing a significant reduction of number of germinal vesicles (GV) as well as metaphase
I (M1) oocytes could reach metaphase II stage in Endometriosis group as compared to controls. iii)Since spindle structure needs to coordinate the alignment as well as normal segregation of homologous chromosomes and sister chromatids in 2 subsequent meiotic division, disruption of meiotic spindle causes abnormal chromosomes alignment and fertilization. Of the 2 methods by which spindle morphology is studied polarized light microscopy overcomes fixing problem of confocal microscopy. Barcelos et al. checked spindle morphology following IVM comparing oocytes obtained from Endometriosis patients $(n=35)$ with oocytes from control group with tubal factor infertility $(n=19)$. Till recently no significant differences were observed. Recently Goud et al. using same IVM, observed a>percentage of abnormal spindles in oocytes obtained from Endometriosis patients as compared to ART secondary to male factor infertility $(66.7 \%$ vs $16 \%, \mathrm{p}<0.05)$. Only study that observed spindle in mature oocytes unlike all earlier ones on IVM ,failed to see any significant changes in Endometriosis patients iv)Cytoplasm of mature oocytes have a very high mitochondrial content as compared to other cell types, since it can contain up to 105 mitochondria. The $1^{\text {st }}$ and only study that examined correlation between cytoplasm ultrastructure of oocytes and Endometriosis presence was conducted by $\mathrm{Xu}$ et al regarding intra cytoplasmic sperm injection (ICSI). Using transmission electron microscopy (TEM) 50MII oocytes from patients with laparoscopically diagnosed minimal/mild Endometriosis control found a >number of Endometriosis patients contained decentralized chromatin with a voluminous nucleolus as compared to control. Further oocytes from patients with Endometriosis had both a > percentage of abnormal mitochondria (containing small/ swollen as well as blurred vacuoles) and in total lower quantity of mitochondrial DNA (mtDNA) copies seen using quantitative PCR. They concluded low mtDNA amount particularly shows reduced oocyte quality with minimal/mild Endometriosis.

Sirtuins are a family of deacetylases which modify structural proteins, metabolic enzymes, and histones for altering cellular protein placement as well as function. In mammals, 7 Sirtuins, that are related to functions like oxidative stress or metabolic homeostasis correlated with increasing age, degeneration/ carcinoma. Gonzalez Fernandez et al. [10] explored gene expression of Sirtuins by qRT-PCR in human mural granulosa-lutein cells (hGL) from IVF subjects in various infertility diagnostic groups and in oocyte donors (OD; Control grp). In Study 1 gene expression levels of Sirtuins as well as association with age as well as IVF parameters in women having no ovarian factor was done. They observed that significantly>expression levels of SIRT1, SIRT2 as well as SIRT5 in patients $\geq 40$ yrs old as compared to OD and in women among 27-39yrs age with tubal/male factor and no ovarian factor (NOF). Only SIRT2, SIRT5 and SIRT7 expression was associated with age. In Study 2 gene expression of Sirtuins in poor responders (PR), Endometriosis (EM)and PCOS was done. In contrast to NOF Controls, they observed $>$, SIRT2 gene expression in all diagnostic groups although SIRT3, SIRT5, SIRT6 and SIRT7 expression was > only in PR. Associated with clinical parameters , SIRT1, SIRT6 and SIRT7 correlated in a positive manner with FSH as well as LH doses 
given to EM patients. The number of mature oocytes obtained in PR correlated in a positive manner with expression amounts of SIRT3, SIRT4, SIRT5. Hence emphasizing on that cellular physiopathology in PR's follicles might be correlated with cumulative DNA damage that points that future studies are required (Figure2); [8]. Since FF surrounds the granulosa cell- oocyte complex and represents one of the modulating factors in the inter cell conversation among the cells within the follicle the substances present in FF are cytokines and immune cells ,that includes IL-6.IL12, sHLA-G, macrophages, NK cells and lymphocytes. These cells as well as cytokines may impact the granulosa cell-oocyte complex, change in immune component contents might be involved in alteration in folliculogenesis, oocyte maturation, oocyte quality as well as ovulation. Moreover, changes in these balances are probably responsible for immune mediated conditions like Endometriosis. Prins et al., gave a detailed evaluation on FF immune function, as well as FF substance alterations in Endometriosis patients. Escalation of macrophages in FF of Endometriosis patients as well as various cytokines have been documented. The part played by particular immune cells in FF and insight about the biological mode in healthy women as well as Endometriosis patients is unknown. More studies in this field will give us greater understanding in the role of FF immune cells and the influence of change in these balances in patients with Endometriosis [9].

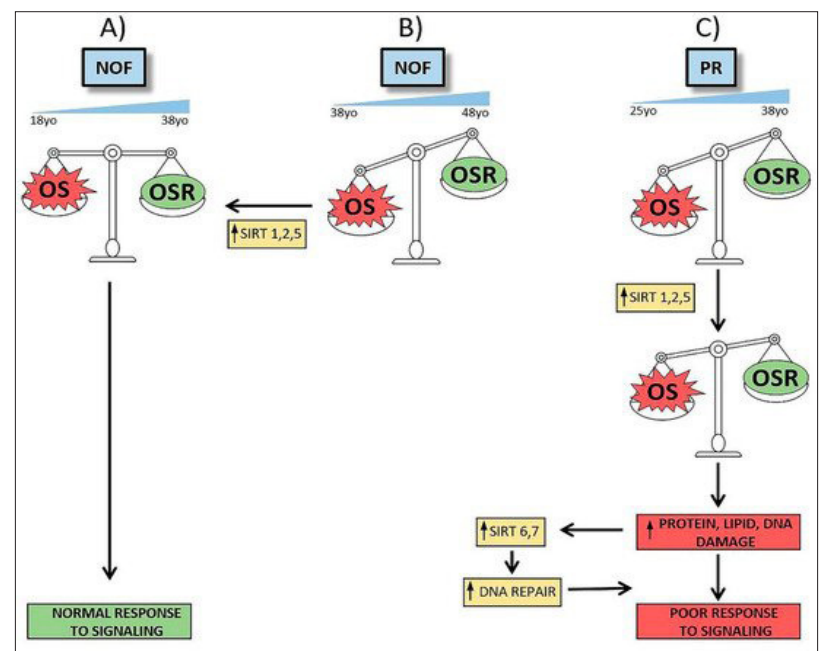

Figure 2: Courtesy ref no.-8-Scheme of alterations in NOF and PR groups and possible Sirtuins' roles. According to our studies, NOF women between 18 to 38 years old may avoid OS damage by OSR, triggering a normal signaling response and maintaining an equilibrated OS/OSR status (A). It is possible that this equilibrium favors OS during pre-menopausal aging (B). In that case, OSR are not sufficient to protect cells from OS actions and SIRT1, SIRT2 and SIRT5 gene expression increase would be required to recover homeostasis. Failing this response, women poor responders show a different sirtuin pattern (C). In this group, women between 25 to 38 years old have an imbalance between OS and OSR similar to older NOF women. Cellular attempts to reach homeostasis by increasing SIRT1, SIRT2 and SIRT5 gene expression are insufficient and it is necessary activate protein, lipid and DNA repair mechanisms and others sirtuins' expression. Despite the fact that SIRT6 and SIRT7 gene expression increase, cells cannot response to signaling and homeostasis cannot be recovered, leading to a clinically poor response to follicle stimulation.

In follicular formation there is key role of granulosa cell [10]. In case of endometriotic subjects, abnormalities of granulosa cell might interfere with oocyte maturation and result in poor oocyte quality [11]. Telomerase was found as a biomarker of the potential that germ cells possess [12]. Premature aging and decreased fecundity were seen in mice having Telomerase deficiency [13]. Earlier Li et al. [14] revealed that telomerase activity (TA) in granulosa cell (GTA), had a positive association with IVF treatment results [14]. Healthy follicles showed $>$ amounts of TA, as compared to decreased GTA =>enhancement of atretic follicles [15]. Subjects having subtle and biochemical premature ovarian insufficiency (POI) further demonstrated reduced TA in their granulosa cells $[16,17]$. Overall these observations point that GTA might work as an ovarian function biomarker.

The effect of ovarian endometriosis on granulose cells was recently studied by $\mathrm{Li}$ et al. [18], that correlates molecular physiology with results in fertility. An efficient oocyte as well as granulosa cell crosstalk taking place the ovarian follicles sees to it that formation of a mature oocyte occurs for obtaining an embryo having full competency resulting in a pregnancy that is viable. $\mathrm{Li}$ et al. [18] described how internally endometriotic lesions present on the ovarian tissue resulted in intrinsic injury by evaluating the inflammatory paths as well as telomerase action within granulosa cell. 80 women got enrolled prospectively having stage II-III Endometriomas as well as 104 controls who were having oocyte recovery utilizing artificial reproductive therapy (ART) therapy. The 2 groups developed pregnancy (clinical)with equal chances, that agrees with most of current literature and helps in giving us assurance with regard to ART overcoming the problems in endometriotic subjects. But emphasis has been paid by them on the impaired results of folliculogenesis which takes place when lesions are existing. 
Actually, the antral follicle count (AFC), luteinizing hormone (LH) amounts as well as the number of oocytes recovered, and mature oocytes all were $<$ in their Endometriomas group. Stimulated by these observations they tried to evaluate the molecular reasons behind this effect on follicle formation via a number of repeated in vitro studies where they separated granulosa cell from the follicular fluid ,evaluated them for any basal inflammatory changes as well as culturing them in the exogenous inflammatory stimulants added for getting a response. Their findings were similar to the common factors in such settings like the nuclear factor (NF) $\kappa B$ as well as tumour necrosis factor alpha $(\mathrm{TNF} \alpha)$ paths that seem to be controlled separately in granulosa cell in endometriotic subjects as compared to controls. Particularly action of NFkB in addition to its 2 helpers IKк $\beta$ as well as IKB $\alpha$ was > in the granulosa cell from the follicular fluid of endometriotic subjects as compared to healthy cells. To find the upstream regulators of $\mathrm{NF \kappa B}$ parts of granulosa cell of endometriotic subjects. Their observation was that TNF $\alpha$ found within the follicular fluid (FF) had a positive association with the expression of NFKB in granulosa cell, a finding which was later confirmed by crucial cell culture studies, where TNF $\alpha$ therapy directly escalated the excess of NFKB as well as IKB $\alpha$. The biggest importance of these experiments was the innovative understanding of the telomerase enzyme action when endometriosis was existent along with its associated inflammation. Recently trying to assess telomere length as well as telomerase activity in granulosa cell of patients with infertility has been on the rise that has turned to be a good marker of oocyte competence. Telomeres represent conserved areas at the distal part of chromosomes which aid in conferring protection to genomic integrity.

Escalated mitotic action of granulosa cell as well as follicular hormones lead to the shortened length of telomeres that has to be restored by telomerase activity. Minimal but essential proof has aided in the understanding that the activity of telomerase is >in healthy as well as small follicles as compared to atretic larger follicles and this activity of enzymes can be enhanced by estrogens [18]. This telomeres length as well as telomerase enzyme activity as far as theoretical explanation is that they represent divergence but separate dynamics got detailed in particular situations ,Like granulosa cell of women having a diagnosis of premature ovarian failure(POF) had shorter telomeres as well as < telomerase activity as compared to control ladies, while absence of normal telomerase activity enhances the chances for women to have POF 11 times [19]. Though experiments of cellular modes with regards to telomere structures within oocytes as well as granulosa cell might aid in unfolding the underlying causes related to infertility, mostly little work has been done in this field.

In $2017 \mathrm{Li}$ et al. [18] utilized the niche, detailing the telomeres length as well as telomerase activity in polycystic ovarian syndrome (PCOS). Their observations were shorter telomeres but the granulosa cell telomerase activity (GTA) was same in PCOS subjects as compared to controls $[19,20]$. In this study, they found that GTA in ovarian endometriotic subjects associated positively with the number of mature oocytes, negatively with granulosa cell NFKB activity and reduced following TNF $\alpha$ therapy. Greater information regarding GTA dynamics was added by studies on (human telomerase reverse transcriptase (hTERT), the catalytic subunit of the telomerase enzyme was reduced with TNF $\alpha$ therapy and enhanced by NFKB inhibitor. An essential observation of this study although negative is that both GTA as well as hTERT were only little lower in endometriotic subjects as compared to controls $(\mathrm{p}=0.16$ and $p=0.55$ respectively) while NF $\kappa B$ inhibitor did not escalate GTA in granulosa cell culture. It needs to be seen if lowering TNF $\alpha$ activity or use of NFкB inhibitor might be of help in re-establishing the hTERT activity needs to be studied further.

On the basis of in vitro findings, they posited a possible mode where lesion led to escalation of FF TNF $\alpha$ stimulates NFKB cascade activation in granulosa cell that in turn decreases telomerase activity and effects physiologic increase in telomeres that is key for folliculogenesis as well as oocyte quality. Important is follicular $\mathrm{TNF} \alpha$ levels was not separate in case as well as controls but the in vivo findings here correlated with limited size sample size or inclusion of earlier operated endometriotic subjects that might have contribute to the complicated nature regarding this. There is requirement for experiments detailing pathophysiology of granulosa cell in endometriotic subjects and time to unveil why subfertility in this disease both for patients as well as professionals [21].

\section{Conclusion}

Thus trying to understand why infertility results in Endometriosis patients lot of efforts have been put in originally most studies concentrated on studying follicular fluid for finding the reason of poor quality of oocytes among Endometriosis patients where researchers observed enhanced oxidative stress as well as ER Stress in cases of Endometriosis subjects.

Later deciding to study oocytes directly researchers observed

1. Altered mitochondrial numbers as well as abnormal mitochondria along with reduced mitochondrial DNA in oocytes of Endometriosis patients

\section{Spindle abnormalities}

3. ZP hardening of oocytes of Endometriosis patients

4. Abnormal spindle patterns and

5. Altered morphology in form of dark central granulation by carrying out study on oocytes during IVM mostly.

Further the role of Sirtuins in effecting the alteration in cytokines in FF associated with Endometriosis patients was emphasized as reflecting which Sirtuins are associated with poor oocyte quality with aging, PR as well as Endometriosis patients and PCOS subjects. Li et al. [18] utilized the niche,detailin $g$ the telomeres length as well as telomerase activity in PCOS subjects where they found shorter telomeres but the granulosa cell telomerase activity (GTA) was same in PCOS subjects found that GTA in ovarian endometriotic subjects associated positively with the number of mature oocytes, negatively with granulosa cell $\mathrm{NF \kappa B}$ activity and reduced following TNF $\alpha$ therapy. Greater information regarding GTA dynamics was added 
by studies on hTERT, the catalytic subunit of the telomerase enzyme was reduced with TNF $\alpha$ therapy and enhanced by NFKB inhibitor .An essential observation of this study although negative ,is that both GTA as well as hTERT were only little lower in endometriotic subjects as compared to controls. On the basis of in vitro findings, they posited a possible mode where lesion led to escalation of FF $\mathrm{TNF} \alpha$ stimulates NFKB cascade activation in granulosa cell that in turn decreases telomerase activity and effects physiologic increase in telomeres that is key for folliculogenesis as well as oocyte quality. Important is follicular TNF $\alpha$ levels was not separate in case as well as controls But the in vivo findings here correlated with limited size sample size or inclusion of earlier operated endometriotic subjects Further work is being needed to find why granulosa cells are involved in Endometriosis patients, studying a larger number of cohort to try to get better possible therapies to reduce oxidative stress and see to it that abnormal cytokines are prevented so that we get optimum oocytes of good quality.

\section{References}

1. Eskenazi B, Warner ML (1997) Epidemiology of endometriosis. Obstet Gynaecol Clin North Am 24(2): 235-258.

2. Meuleman C, Vandenabeele B, Fleuws S, Spiessens C, Timmerman D, et al. (2009) High prevalence of endometriosis in infertile women with normal ovulation and normospermic pattern. Fertil Steril 92(1): 68-74.

3. Scutiero G, Iannone P, Bernardi G, Bonaccorsi G, Spadaro S, et al. (2017) Oxidative stress and endometriosis: A Systematic Review of the Literature. Oxid Med Cell Longer 2017: 7265238.

4. Pellicer A, Albert C, Mercader A, Musoles BF, Remohi J, et al. (1998) The follicular and endocrine environment in women with Endometriosis: Local and Systemic cytokine production. Fertil Steril 70(3): 425-431.

5. Avila J, Fernandez GR, Rotoli D, Hernandez J, Palumbo A (2016) Oxidative stress in granulosa-lutein cells from in vitro fertilization patients. Reprod Sci 23(12): 1656-1661.

6. Kunitomi C, Harada M, Takahashi N, Azhary JMK, Kusamoto A, et al (2019) Activation of endoplasmic reticulum stress mediates oxidative stress-induced apoptosis of granulosa cells in ovaries affected by endometrioma. Mol Hum Reprod.

7. Sanchez AM, Stella Vani V, Bartiromo L, Papaleo E, Zilberberg E, et al. (2017) Is the oocyte quality affected by Endometriosis? A Review of the literature. J Ovarian Res 10(1): 43.

8. Prins JR, Marissen LM, Scherjon SA, Hoek A, Cantineau A (2012) Is there an immune modulating role of follicular fluid in Endometriosis? A narrative reviews. Reproduction.

9. Madtuda F, Inoue N, Manabe N, Ohkura S (2012) Follicular growth and atresia in mammalian ovaries: Regulation by survival and death of granulosa cells. J Reprod Dev 58(1): 44-50.

10. Fernandez GR, Ramirez MR, Rotoli D, Hernandez J, Naftolin F, et al. (2019) Granulosa lutein cell sirtuins gene expression profiles differ between normal donors and infertile women. Int J Mol Sci 21(1).

11. Saito H, Seino T, Kaneko T, Nakahara K, Toya M, etal. (2002) Endometriosis and oocyte quality. Gynaecol Obstet Invest 53(Suppl 1): 46-51.

12. Ozturk S, Sozen B, Demir N (2014) Telomere length and telomerase activity during oocyte maturation and early embryo development in mammalian species. Mol Human Reprod 20(1): 15-30.

13. Liu L, Blasco M, Trimarchi J, Keefe D (2002) An essential role for functional telomeres in mouse germ cells during fertilization and early development. Dev Biol 249: 74-84.

14. Li Y, Dieng B, Ouyang N, Yuan P, Wang W (2017) Telomere length is short in PCOS and oral contraceptive does not affect telomerase activity in granulosa cells of patients with PCOS. J Assist Reprod Genet 34(7): 849859.

15. Chen H, Wang W, Mo Y, Ma Y, Ouyang N, et al. (2011) Women with high telomerase activity in luteinized granulosa cell having a higher pregnancy rate during in vitro fertilization treatment. J Assist Reprod Genet 28: 797-807.

16. Yamagata Y, Nakamura Y, Umayahara K, Harada A, Takayama H, et al. (2002) Changes in telomerase activity in experimentally induced atretic follicles of immature rats. Endocrine J 49(6): 589-595.

17. Xu X, Chen X, Zhang X, Liu Y, Wang Z, et al. (2017) Impaired telomere length and telomerase activity in peripheral blood leukocytes and granulosa cells in patients with biochemical primary ovarian insufficiency. Human Reprod 32(1): 201-207.

18. Li Y, Li R, Ouyang N, Dai K, Yuan P, et al. (2019) Investigating the impact of local inflammation on granulosa cells and Follicular development in women with ovarian Endometriosis. Fertil Steril 112(5): 882-891.

19. Kosebent EG, Uysal F, Ozturk S (2018) Telomere length and telomerase activity during Folliculogenesis in mammals. J Reprod Dev 64(6): 477484 .

20. Butts S, Riethman H, Ratcliffe S, Shaunik A, Coutifaris C, et al. (2009) Correlation of telomere length and telomerase activity with occult ovarian insufficiency. J Clin Endocrinol Metab 94(12): 4835-4843.

21. Vigano P, Makieva S (2019) Lifting the Endometrioma veil on granulosa cells. Fertil Steril 112(5): 813-814.

For possible submissions Click below:

Submit Article 